Nevşehir Bilim ve Teknoloji Dergisi Cilt 3(2) 66-73 2014

DOI: 10.17100/nevbiltek.210931

URL: http://dx.doi.org/10.17100/nevbiltek.210931

\title{
An Application of Krasnoselskii Fixed Point Theorem to Some Nonlinear Functional Integral Equations
}

\author{
Ümit ÇAKAN ${ }^{1, *}$, İsmet ÖZDEMIR ${ }^{2}$ \\ ${ }^{1}$ Nevşehir Hacı Bektaş Veli Üniversitesi, Fen Edebiyat Fakültesi, Matematik Bölümü, Nevşehir \\ ${ }^{2}$ Inönü Üniversitesi, Eğitim Fakültesi, Malatya
}

Abstract

The purpose of this paper is to study the existence of solutions of some nonlinear functional integral equations in the space of continuous functions defined on interval $[0, a]$ under some conditions. To do this, we will use Krasnoselskii Fixed Point Theorem and Ascoli-Arzela Theorem. Finally we will give an example to illustrate our result.

Keywords: Nonlinear Integral equations, Krasnoselskii Fixed Point Theorem, Ascoli-Arzela Theorem.

\section{Krasnoselskii Sabit Nokta Teoreminin Lineer Olmayan Bazı Fonksiyonel İntegral Denklemlere Uygulaması}

Özet

Bu çalışmanın amacı $[0, a]$ aralığı üzerinde tanımlı ve sürekli fonksiyonların uzayında, bazı kabuller altında lineer olmayan bazı fonksiyonel integral denklemlerin çözümlerinin varlığını araştırmaktır. Bunun için Krasnoselskii Sabit Nokta Teoremini ve AscoliArzela Teoremini kullanılacaktır. Son olarak elde edilen sonuçları açıklayıcı bir örnek verilecektir.

Anahtar Kelimeler: Lineer Olmayan İntegral Denklemler, Krasnoselskii Sabit Nokta Teoremi, Ascoli-Arzela Teoremi.

*e-mail: umitcakan@nevsehir.edu.tr 


\section{Introduction}

Many nonlinear problems arising from the most areas of natural sciences such as engineering, mechanics, physics, economics and so on can be represented under the mathematical point of view. Especially nonlinear integral equations are often used in characterization of these problems. For example quadratic integral equations used in many applications in the theory of radioactive transfer, in the theory of neutron transport and in the kinetic theory of gases, [1]. Some nonlinear problems involve the study of solutions of nonlinear operator equations of the form

$$
A x+B x=x, \quad x \in S
$$

where $S$ is a closed, bounded and convex subset of a Banach space $X$ and $A, B$ are two operators defined on $\mathrm{S},[2]$.

The author considered the following equation in [3]

$$
x(t)=f(t, x(\alpha(t))) \int_{0}^{1} u(t, s, x(s)) d s,
$$

for $t \in[0,1]$.

In 2004, J. Banaś and A. Martinon [4] studied the nonlinear quadratic integral equation of Volterra type having the form

$$
x(t)=b(t)+x(t) \int_{0}^{t} u(t, s, x(s)) d s, t \in[0, T] .
$$

Then K. Maleknejad et al. [5] studied the existence of solutions of the following equation

$$
x(t)=g(t, x(\beta(t))) \int_{0}^{1} u(t, s, x(s)) d s, t \in[0,1] .
$$

Also the authors $[6,7]$ dealt with the following equation in space $C[0, a]$

$$
x(t)=f(t, x(\alpha(t)))+g(t, x(\beta(t))) \int_{0}^{\varphi(t)} u(t, s, x(\gamma(s))) d s
$$

and give the following conditions in [7].

$\left(a_{1}\right)$ The functions $\alpha, \beta: I \rightarrow I, \varphi: I \rightarrow \mathbb{R}_{+}$and $\gamma: \mathbb{R}_{+} \rightarrow \mathbb{R}_{+}$are continuous.

$\left(a_{2}\right)$ The functions $f, g: I \times \mathbb{R} \rightarrow \mathbb{R}$ are continuous and there exist the positive constants $k$ and $l$ such that

$$
\begin{gathered}
\left|f\left(t, x_{1}\right)-f\left(t, x_{2}\right)\right| \leq k\left|x_{1}-x_{2}\right| \\
\left|g\left(t, x_{1}\right)-g\left(t, x_{2}\right)\right| \leq l\left|x_{1}-x_{2}\right|
\end{gathered}
$$

for all $t \in I$ and $x_{1}, x_{2} \in \mathbb{R}$.

$\left(a_{3}\right)$ The function $u: I \times[0, T] \times \mathbb{R} \rightarrow \mathbb{R}$ is continuous and there exist the positive constants $m, n$ and $p$ such that

$$
|u(t, s, x)| \leq m+n|x|^{p}
$$

for all $t \in I, s \in[0, T]$ and $x \in \mathbb{R}$.

$\left(a_{4}\right)$ The inequality

$$
M+T(m+n)(l+N)+k<1
$$

is held, where $M$ and $N$ are positive constants such that $|g(t, 0)| \leq N$ and $|f(t, 0)| \leq M$ for all $t \in I$. 
In this paper, we will consider again Eq. (3) for $t \in[0, a]$. In Section 2, we present some definitions and preliminaries such as modulus continuity, equicontinuity, Ascoli-Arzela Theorem and so on. In Section 3, we give our main result concerning the existence of solutions of the integral equation (3) by applying Krasnoselskii Fixed Point Theorem defined by M. A. Krasnoselskii [8] and finally we establish an example to show that our result is applicable but the some previous ones are not.

\section{Definition and Auxiliary Facts}

In this section, we give some definitions and theorems which will be needed next section. Let $(X,\|\cdot\|)$ be an infinite Banach space with zero element $\theta$. We write $B(x, r)$ to denote the closed ball centered at $x$ with radius $r$ and especially we write $B_{r}$ in case of $x=\theta$.

Definition 2.1 [9] Let $U$ be a compact subset of $\mathbb{R}$ and $C(U)$ denote the family of all continuous functions from $U$ to $\mathbb{R}$. Then modulus continuity of $x \in C(U)$ is the function $\omega(x,):.[0, \infty) \rightarrow[0, \infty)$ defined by

$$
\omega(x, \varepsilon)=\sup \left\{\left|x\left(t_{1}\right)-x\left(t_{2}\right)\right|: t_{1}, t_{2} \in U \text { and }\left|t_{1}-t_{2}\right| \leq \varepsilon\right\}
$$

for $\varepsilon>0$.

Definition 2.2 [10] Let $X$ be an arbitrary nonempty set and $E$ be a family of complex valued functions defined on $X$. We call $E$ uniformly bounded if there exists a real number $M$ such that

$$
|x(t)| \leq M
$$

for every $x \in E$ and $t \in X$.

Definition 2.3 [11] Let $X$ and $Y$ be two metric spaces, and $E$ a family of functions from $X$ to $Y$. The family $E$ is equicontinuous at a point $t_{0} \in X$ if for every $\varepsilon>0$, there exists a $\delta>0$ such that

$$
d_{Y}\left(x\left(t_{0}\right), x(t)\right)<\varepsilon
$$

for all $x \in E$ and all $t \in X$ such that $d_{X}\left(t_{0}, t\right)<\delta$. The family $E$ is equicontinuous if it is equicontinuous at each point of $X$. The family $E$ is uniformly equicontinuous if for every $\varepsilon>0$, there exists a $\delta>0$ such that

$$
d_{Y}\left(x\left(t_{1}\right), x\left(t_{2}\right)\right)<\varepsilon
$$

for all $x \in E$ and all $t_{1}, t_{2} \in X$ such that $d_{X}\left(t_{1}, t_{2}\right)<\delta$.

Theorem 2.1 (Ascoli-Arzela Theorem) Let $\Omega$ be a compact Hausdorff metric space. Then $M \subset C(\Omega)$ is relatively compact if and only if $M$ is uniformly bounded and uniformly equicontinuous.

In 1958, M. A. Krasnoselskii [8] established following theorem which is one of the important results in fixed point theorems.

Theorem 2.2 (Krasnoselskii Fixed Point Theorem) Let $S$ be a closed, bounded and convex subset of a real Banach space $X$ and let $U_{1}$ and $U_{2}$ be operators on $S$ satisfying the following conditions:

(i) $U_{1}(S)+U_{2}(S) \subset S$,

(ii) $U_{1}$ is continuous on $S$ and $U_{1}(S)$ is a relatively compact subset of $X$,

(iii) $U_{2}$ is a strict contraction on $S$, i.e., there exists $k \in[0,1)$ such that

$$
\left\|U_{2}(x)-U_{2}(y)\right\| \leq k\|x-y\|
$$

for every $x, y \in S$.

Then there exists $x \in S$ such that $U_{1} x+U_{2} x=x$. 


\section{Main Result}

In this section we give a theorem for solvability of Eq. (3) under following assumptions. Also we write $I$ to denote the interval $[0, a]$ throughout this section.

(a) The functions $\alpha, \beta: I \rightarrow I, \varphi: I \rightarrow \mathbb{R}_{+}$and $\gamma: \mathbb{R}_{+} \rightarrow \mathbb{R}_{+}$are continuous.

Remark 3.1 Note that assumption (a) implies that there exists a positive constant $\mathrm{T}$ such that

$$
\phi(t) \leq T
$$

for all $t \in I$.

(b) The functions $f, g: I \times \mathbb{R} \rightarrow \mathbb{R}$ are continuous and there exist positive constants $k$ and $l$ such that

$$
\begin{gathered}
\left|f\left(t, x_{1}\right)-f\left(t, x_{2}\right)\right| \leq k\left|x_{1}-x_{2}\right|, \\
\left|g\left(t, x_{1}\right)-g\left(t, x_{2}\right)\right| \leq l\left|x_{1}-x_{2}\right|
\end{gathered}
$$

for all $t \in I$ and $x_{1}, x_{2} \in \mathbb{R}$.

Remark 3.2 Note that assumption (b) implies that there exist positive constants $M$ and $N$ such that

$$
\begin{gathered}
|f(t, 0)| \leq M, \\
|g(t, 0)| \leq N
\end{gathered}
$$

for all $t \in I$.

(c) The function $u: I \times[0, T] \times \mathbb{R} \rightarrow \mathbb{R}$ is continuous. Moreover, there exist a function $h$ : $\mathbb{R}_{+} \rightarrow \mathbb{R}_{+}$which is nondecreasing on $\mathbb{R}_{+}$such that

$$
|u(t, s, x)| \leq h(|x|)
$$

for all $t \in I, s \in[0, T]$ and $x \in \mathbb{R}$.

(d) There exists a positive solution $r_{0}$ of the inequality

$$
k r+M+T(l r+N) h(r) \leq r .
$$

Lemma 3.1 Let $S=B_{r_{0}} \subset C(I)$. We define operator $U_{1}$ on $S$ such that for any $x \in S$

$$
\left(U_{1} x\right)(t)=g(t, x(\beta(t))) \int_{0}^{\varphi(t)} u(t, s, x(\gamma(s))) d s .
$$

Then operator $U_{1}$ is continuous on $S$ under above assumptions.

Proof. For $\varepsilon>0$ and any $x, y \in B_{r_{0}}$ such that $\|x-y\| \leq \varepsilon$ we get

$$
\begin{aligned}
& \left|\left(U_{1} x\right)(t)-\left(U_{1} y\right)(t)\right| \\
& =\left|g(t, x(\beta(t))) \int_{0}^{\varphi(t)} u(t, s, x(\gamma(s))) d s-g(t, y(\beta(t))) \int_{0}^{\varphi(t)} u(t, s, y(\gamma(s))) d s\right| \\
& \leq\left|g(t, x(\beta(t))) \int_{0}^{\varphi(t)} u(t, s, x(\gamma(s))) d s-g(t, y(\beta(t))) \int_{0}^{\varphi(t)} u(t, s, x(\gamma(s))) d s\right| \\
& +(|g(t, y(\beta(t)))-g(t, 0)|+|g(t, 0)|) \int_{0}^{\varphi(t)}|u(t, s, x(\gamma(s)))-u(t, s, y(\gamma(s)))| d s \\
& \leq l|x(\beta(t))-y(\beta(t))| T h\left(r_{0}\right)+(l|y(\beta(t))|+N) T \omega_{u_{3}}(I, \varepsilon) \\
& \leq T l\|x-y\| h\left(r_{0}\right)+T(l\|y\|+N) \omega_{u_{3}}(I, \varepsilon) \\
& \leq T\left(l \varepsilon h\left(r_{0}\right)+\left(\operatorname{lr}_{0}+N\right) \omega_{u_{3}}(I, \varepsilon)\right)
\end{aligned}
$$


where

$$
\omega_{u_{3}}(I, \varepsilon)=\sup \left\{|u(t, s, x)-u(t, s, y)|: t \in I, s \in[0, T], x, y \in\left[-r_{0}, r_{0}\right] \text { and }|x-y| \leq \varepsilon\right\} .
$$

Due to the function $u=u(t, s, x)$ is uniformly continuous on the compact set $I \times[0, T] \times\left[-r_{0}, r_{0}\right]$, we infer that $\omega_{u_{3}}(I, \varepsilon) \rightarrow 0$ as $\varepsilon \rightarrow 0$. Hence, the above estimate prove that the operator $U_{1}$ is continuous on $S$.

Lemma 3.2 Under assumptions (a)-(d) $U_{1}(S)$ is a relatively compact subset of $C(I)$.

Proof. The main tool used in this proof is Ascoli-Arzela Theorem. Let consider $\varepsilon>0$, any $U_{1} x \in U_{1}(S)$ and any $t_{1}, t_{2} \in I$ such that $\left|t_{1}-t_{2}\right| \leq \varepsilon$. Then we obtain the following inequalities by using above conditions

$$
\begin{aligned}
& \left|\left(U_{1} x\right)\left(t_{1}\right)-\left(U_{1} x\right)\left(t_{2}\right)\right| \\
& =\left|g\left(t_{1}, x\left(\beta\left(t_{1}\right)\right)\right) \int_{0}^{\varphi\left(t_{1}\right)} u\left(t_{1}, s, x(\gamma(s))\right) d s-g\left(t_{2}, x\left(\beta\left(t_{2}\right)\right)\right) \int_{0}^{\varphi\left(t_{2}\right)} u\left(t_{2}, s, x(\gamma(s))\right) d s\right| \\
& \leq\left|g\left(t_{1}, x\left(\beta\left(t_{1}\right)\right)\right) \int_{0}^{\varphi\left(t_{1}\right)} u\left(t_{1}, s, x(\gamma(s))\right) d s-g\left(t_{1}, x\left(\beta\left(t_{2}\right)\right)\right) \int_{0}^{\varphi\left(t_{1}\right)} u\left(t_{1}, s, x(\gamma(s))\right) d s\right| \\
& +\left|g\left(t_{1}, x\left(\beta\left(t_{2}\right)\right)\right) \int_{0}^{\varphi\left(t_{1}\right)} u\left(t_{1}, s, x(\gamma(s))\right) d s-g\left(t_{2}, x\left(\beta\left(t_{2}\right)\right)\right) \int_{0}^{\varphi\left(t_{1}\right)} u\left(t_{1}, s, x(\gamma(s))\right) d s\right| \\
& +\left|g\left(t_{2}, x\left(\beta\left(t_{2}\right)\right)\right) \int_{0}^{\varphi\left(t_{1}\right)} u\left(t_{1}, s, x(\gamma(s))\right) d s-g\left(t_{2}, x\left(\beta\left(t_{2}\right)\right)\right) \int_{0}^{\varphi\left(t_{1}\right)} u\left(t_{2}, s, x(\gamma(s))\right) d s\right| \\
& =\left|g\left(t_{1}, x\left(\beta\left(t_{1}\right)\right)\right)-g\left(t_{1}, x\left(\beta\left(t_{2}\right)\right)\right)\right| \int_{0}^{\varphi\left(t_{1}\right)}\left|u\left(t_{1}, s, x(\gamma(s))\right)\right| d s \\
& +\left|g\left(t_{1}, x\left(\beta\left(t_{2}\right)\right)\right)-g\left(t_{2}, x\left(\beta\left(t_{2}\right)\right)\right)\right| \int_{0}^{\varphi\left(t_{2}\right)}\left|u\left(t_{1}, s, x(\gamma(s))\right)\right| d s \\
& \times\left(\int_{0}^{\varphi\left(t_{2}\right)} \varphi\left(t_{1}\right)\right. \\
& \left.\leq \mid l \omega(x, \omega(\beta, \varepsilon))+\omega_{g}(I, \varepsilon)\right] T h\left(r_{0}\right)+\left(l r_{0}+N\right)\left[T \omega_{u_{1}}\left(I_{1}, \varepsilon\right)+\omega(\varphi, \varepsilon) h\left(r_{0}\right)\right], \\
& \left.+\left|g\left(t_{2}, x\left(\beta\left(t_{2}\right)\right)\right)\right| \mid \int_{0}^{\varphi\left(t_{1}\right)} u\left(t_{1}, s, x(\gamma(s))\right) d s+\int_{0} u\left(t_{1}, s, x(\gamma(s))\right) d s-x(\gamma(s))\right) d s \mid \\
& +\left(l\left|x\left(\beta\left(t_{1}\right)\right)-x\left(\beta\left(t_{2}\right)\right)\right|+\int_{g}(I, \varepsilon)\right) \int_{0}^{\varphi\left(t_{1}\right)}\left|u\left(t_{1}, s, x(\gamma(s))\right)\right| d s \\
& \left.+\left(t_{2}, x\left(\beta\left(t_{2}\right)\right)\right)-g\left(t_{2}, 0\right)|+| g\left(t_{2}, 0\right) \mid\right) \times
\end{aligned}
$$

where

$$
\omega\left(x_{i}, \varepsilon\right)=\sup \left\{\left|x_{i}\left(t_{1}\right)-x_{i}\left(t_{2}\right)\right|: t_{1}, t_{2} \in I \text { and }\left|t_{1}-t_{2}\right| \leq \varepsilon\right\},
$$

for $i=1,2,3$ such that $x_{1}=x, x_{2}=\beta$ and $x_{3}=\varphi$.

Also 


$$
\begin{gathered}
\omega_{g}(I, \varepsilon)=\sup \left\{\left|g\left(t_{1}, x\right)-g\left(t_{2}, x\right)\right|: t_{1}, t_{2} \in I, x \in\left[-r_{0}, r_{0}\right] \text { and }\left|t_{1}-t_{2}\right| \leq \varepsilon\right\}, \\
\omega_{u_{1}}(I, \varepsilon)=\sup \left\{\left|u\left(t_{1}, s, x\right)-u\left(t_{2}, s, x\right)\right|: t_{1}, t_{2} \in I, s \in[0, T], x \in\left[-r_{0}, r_{0}\right] \text { and }\left|t_{1}-t_{2}\right| \leq \varepsilon\right\} .
\end{gathered}
$$

Hence we write $\omega(\beta, \varepsilon) \rightarrow 0, \omega(x, \varepsilon) \rightarrow 0, \omega(\varphi, \varepsilon) \rightarrow 0$ as $\varepsilon \rightarrow 0$ the from above estimate by using uniformly continuity of these functions on the set $I$. Similarly, we get $\omega_{g}(I, \varepsilon)$ and $\omega_{u_{1}}(I, \varepsilon) \rightarrow 0$ as $\varepsilon \rightarrow 0$ since functions $\mathrm{g}$ and $\mathrm{u}$ are uniformly continuous on the sets $I \times\left[-r_{0}, r_{0}\right]$ and $I \times[0, T] \times$ $\left[-r_{0}, r_{0}\right]$ respectively. On the other hand for any $x \in U_{1}(S)$ and all $t \in[0, a]$ we have $|x(t)| \leq\|x\| \leq$ $r_{0}$. This means that the set $U_{1}(S)$ is uniformly bounded and uniformly equicontinuous. So $U_{1}(S)$ is a relatively compact subset of $C(I)$ from Acoli-Arzela Theorem.

Theorem 3.1 Under assumptions (a)-(d), Eq. (3) has at least solution $x=x(t)$ which belongs to $B_{r_{0}} \subset C[0, a]$.

Proof. Note that we will use Krasnoselskii Fixed Point Theorem as our main tool. We define operator $U_{2}$ on $S$ such that $\left(U_{2} x\right)(t)=f(t, x(\alpha(t)))$, for any $x \in S$.

Using the conditions (a)-(d) we have

$$
\begin{aligned}
\left|\left(U_{2} x\right)(t)-\left(U_{2} y\right)(t)\right| & =|f(t, x(\alpha(t)))-f(t, y(\alpha(t)))| \\
& \leq k|x(\alpha(t))-y(\alpha(t))| \\
& \leq k\|x-y\|
\end{aligned}
$$

for any $x, y \in S$, where $k \in(0,1)$ from (4). So we infer that $U_{2}$ is a strict contraction on $S$. Also for any $x \in S$,

$$
\begin{aligned}
\left|\left(U_{1} x\right)(t)+\left(U_{2} x\right)(t)\right| & =\left|f(t, x(\alpha(t)))+g(t, x(\beta(t))) \int_{0}^{\varphi(t)} u(t, s, x(\gamma(s))) d s\right| \\
& \leq|f(t, x(\alpha(t)))-f(t, 0)|+|f(t, 0)| \\
& +(|g(t, x(\beta(t)))-g(t, 0)|+|g(t, 0)|) \int_{0}^{\varphi(t)}|u(t, s, x(\gamma(s)))| d s \\
& \leq k r_{0}+M+T\left(\operatorname{lr}_{0}+N\right) h\left(r_{0}\right) \\
& \leq r_{0} .
\end{aligned}
$$

Then $U_{1}(S)+U_{2}(S) \subset S$. Hence $U_{1}$ and $U_{2}$ are satisfy Krasnoselskii conditions. Consequently, there exists at least one function $x=x(t) \in C[0, a]$ such that $\left(U_{1} x\right)(t)+\left(U_{2} x\right)(t)=x(t)$. This completes the proof.

Remark 3.3 Function $h:[0, \infty) \rightarrow[0, \infty)$ defined by $h(x)=m+n x^{p}$ is nondecreasing for any positive fixed $p$. Also, if condition $\left(a_{4}\right)$ in [7] satisfies, that is,

$$
M+T(m+n)(l+N)+k<1,
$$

then condition (d) is held. This means that Theorem 3.1 is applicable to all equations in [7] but the converse of this may not be correct. Now, we will give an example to illustrate this case.

Example 3.1 We will deal with the following integral equation in $C[0,1]$ : 


$$
x(t)=\frac{1-\cos t}{10}+\frac{x(t)}{8}+\frac{1+x(\sqrt{t})}{7+t} \int_{0}^{t}\left(t^{2} s+x^{2}(s)+\ln \left[\frac{1+\exp x(s)}{2}\right]\right) d s .
$$

Put

$$
\begin{gathered}
\beta(t)=\sqrt{t}, \quad \alpha(t)=\varphi(t)=t, \quad \gamma(s)=s, \\
f(t, x)=\frac{1-\cos t}{10}+\frac{x}{8}, \quad g(t, x)=\frac{1+x}{7+t}, \\
u(t, s, x)=t^{2} s+x^{2}+\ln \left[\frac{1+\exp x}{2}\right]
\end{gathered}
$$

and

$$
h(r)=1+r^{2}+\ln \left[\frac{1+\text { expr }}{2}\right], \quad T=1, \quad l=N=\frac{1}{7}, \quad k=\frac{1}{8}, \quad M=\frac{1}{10} .
$$

Since $r_{0}=1$ is a solution of

$$
\frac{r}{8}+\frac{1}{10}+\left(\frac{r+1}{7}\right)\left(1+r^{2}+\ln \left[\frac{1+\text { expr }}{2}\right]\right) \leq r,
$$

(d) is satisfied. So, from Theorem $3.1 \mathrm{Eq}$. (5) has at least one solution $x=x(t) \in B_{1} \subset C[0,1]$.

Since there is not constant $m, n$ and $p$ such that

$$
|u(t, s, x)| \leq m+n|x|^{p},
$$

for all $t, s \in[0,1]$ and $x \in \mathbb{R}$, the previous results presented in [5] and [7] are inapplicable to integral equation (5).

\section{Acknowledgment}

The authors would like to thanks the referees for their helpful comments and suggestions on the improvement of this paper.

\section{References}

[1] Hu S., Khavanin M. and Zhuang W., "Integral equations arising in the kinetic theory of gases", Aplpicable Analysis, 34, 261-266, 1989.

[2] Krasnoselskii M. A., "Topological Methods in the Theory of Nonlinear Integral Equations", Pergamon Press, New York, 1964.

[3] Deimling K., "Nonlinear Functional Analysis”, Springer, Berlin, 1985.

[4] Banaś J. and Martinon A., "Monotonic solutions of a quadratic integral equation of Volterra type”, Computers \& Mathematics with Applications, 47, 271-279, 2004.

[5] Maleknejad K., Nouri K. and Mollapourasl R., "Investigation on the existence of solutions for some nonlinear functional-integral equations”, Nonlinear Analysis, 71, 1575-1578, 2009.

[6] Çakan Ü. and Özdemir İ., "An application of the measure of noncompactness to some nonlinear functional integral equations in $C[0, a]$ ", Advences in Mathematical Sciences and Applications, 23(2), 575-584, 2013.

[7] Özdemir İ., Çakan Ü. and İlhan B., "On the existence of the solutions forsSome nonlinear Volterra integral equations”, Abstract and Applied Analysis, 2013, Article ID 689234, 5 pages. 
[8] Krasnoselskii M. A., "Some problems of nonlinear analysis”, American Mathematical Society Translations, Series 2, 10(2), 345-409, 1958.

[9] Banaś J. and Goebel K., "Measures of Noncompactness in Banach Space, Lecture Notes in Pure and Applied Mathematics, Vol. 60”, Dekker, New York, 1980.

[10] Ma Tsoy-Wo, “Banach-Hilbert Spaces, Vector Measures and Group Representations”, WorldScientific, Singapore, 2002.

[11] Reed M. and Simon B., “Functional Analysis”, Academic Press, Boston, 1980. 